\title{
Matter (Pudgalastikaya or Pudgala) in Jain Philosophy
}

\author{
Narayan Lal Kachhara \\ Deemed University
}

\begin{abstract}
Pudgalastikaya is one of the six constituent dravyas of loka in Jainism and is the only substance that is sense perceptible. The sense attributes of pudgala are colour, taste, smell, and touch properties which become the basis of its diversity of forms and structures. The smallest constituent of pudgala is paramanu; the other forms are its combinations. The combination of parmanus forms various states of the matter. The paper describes different types of combinations and modes, rules for combinations and properties of aggregates known as vargana. Some varganas associate with the soul and form various types of bodies of organisms and others exist as forms of matter in loka (universe). The paramanu defines the smallest units of energy, space, time, and sense quality of pudgala. Pudgala exists in visible and invisible forms but anything that is visible is definitely pudgala. Pudgala is classified in various ways; one of them is on the basis of touch property and there are pudgalas having two touches, four touches, and eight touches, each class having some specific character that differentiates them in respect of stability and motion. Pudgala is also classified as living, prayoga-parinat, and non-living, visrasa-parinat. The living matter existing as bodies of organisms exhibits some properties that are not found in non-living matter. Modern science has no such distinction which has become a cause of confusion in recognizing the existence of soul. The description of body remains incomplete without considering the presence of soul in the body. In modern terms, a paramanu is a vibrating and moving charge that is bosonic in character. The two-touch and four-touch pudgala do not appear to follow the speed limits prescribed by Special Theory of Relativity. Jain canonical works describe two types of motions sparshad type and asparshad type and these determine the motions of different class of pudgala and soul. Jain philosophy describes the dynamics and motion of parmanu in detail. The paper describes the Jain concepts of matter in detail and compares with the modern concepts to highlight the strength of Jain views. Modern science has explored the properties and behavior of matter in great detail but still there are many concepts that Jain philosophy has to offer.
\end{abstract}

Keywords: Pudgala, matter, paramanu, dravya, charge, vargana (energy fields), subtle cosmos, motion

\section{1. "Matter" in Jainism}

Matter has been studied in Jain Philosophy and by every system of Indian Philosophy. According to Jain metaphysics, one form of Ajivadravya (Non-living substance) is pudgalastikaya (matter substance) which exists in the universe in various forms such as earth, water, fire, air, shadow, objects of four senses-hearing, smell, taste, and touch, physical mind, speech, bodies, etc., up to karmic matter and paramanu (ultimate particle). Pudgala is tangible reality within the sensuous and super sensuous experiences in perceptible and imperceptible conditions. Pudgala is permanent, non-living, non-conscious, extensive, physical, corporeal and

Narayan Lal Kachhara, Dr., Professor Emeritus, Department of Jainology and Comparative Religion \& Philosophy, Jain Vishva Bharati Institute, Deemed University, Ladnun, India; main research field: Jain Philosophy and Science. 
concrete, active, disintegrating and integrating, and changeable substance. ${ }^{1}$ It is characterized by origination, decay and permanence without giving up its essential nature of existence.

Pudgala is the only substance which is murta (corporeal) and perceivable. Rupatva (form)/murtatva (corporeality) or sensory perceptibility is the sum total of the four sensuous qualities as follows. ${ }^{2}$

(1) Colour-five types of primary colour: black, blue, red, yellow, and white;

(2) Taste-five types of taste: sweet, bitter, pungent, sour and astringent;

(3) Smell- - two types of odour: good smell and bad smell;

(4) Touch—eight types of touch: cold, hot, smooth (positive charge), rough (negative charge), light, heavy, soft, and hard.

All colours, tastes, and smells can vary in magnitude and range.

Based on above qualities, the matter substance in nature is of three types. ${ }^{3}$

(1) Matter substance having one colour, one smell, one taste, and two touches;

(2) Matter substance having five colours, two smells, five tastes, and four touches;

(3) Matter substance having five colours, two smells, five tastes, and eight touches.

Paramanu is the two-touch matter substance (pudgala); it has only one colour, one smell, and one taste. ${ }^{4}$ The four-touch pudgala comprise the subtle (suksama) class of matter substance, as aggregates (skandha), which has substantial energy. This matter has five colours, two smells, and five tastes. On the other hand, the eight-touch matter constitutes the gross (badar) class of aggregates comprising of energy and matter. These aggregates have the five colours, two smells, and five tastes. Thus, according to Jaina, all aggregates, containing a large number of paramanus, necessarily possess all colours, smells, and tastes. Generally only one or a few of the colours, smells, and tastes manifest in gross state at a time, the others remain un-manifest. The manifestation of colours, etc., is dependent on the mode of the substance. Some attributes manifest in the natural mode while some other attributes manifest in the alienated modes. The manifestations are both intrinsic and extrinsic. For example, some colours, smells, and tastes manifest in a fruit in the green state and other colours, smells, and tastes manifest in the ripen state.

Cold, hot, smooth, and rough are primary touch qualities of pudgala. The smooth touch is also regarded as positive charge and the rough touch is regarded as negative charge. The other four touch qualities viz. light, heavy, soft, and hard are secondary touch qualities. These touch qualities are supposed to develop when bonding between infinite paramanus produces a gross aggregate. If number of negative paramanus is more in the bonding process, the aggregate contains light touch quality and if positive paramanus are more than heavy, touch is produced in the aggregate. When positive paramanus are in majority and they bond in cold condition, soft touch is produced and when a majority of negative paramanus bond in hot condition, hard touch is produced in the aggregate. ${ }^{5}$ The weight (or mass?) of the aggregate is said to relate to the light and heavy touch qualities. The four touch aggregates and paramanu are weight less. The weight is a property of gross aggregates having eight touches. ${ }^{6}$ This aspect is further discussed below.

In the true sense, the paramanu and aggregates as a class have no beginning; they have always been in existence. But a particular aggregate or paramanu has a beginning and a life. The minimum life of a paramanu as free paramanu and that of an aggregate can be one "samaya" (samaya is the smallest indivisible unit of time and is the time taken by a paramanu moving at slowest speed to move a distance of one pradesa. A pradesa is the space occupied by one paramanu) and maximum life can be innumerable "samayas."7 Thereafter they undergo change. The pudgala are of two types, subtle, and gross, as said earlier. The subtle does not remain 
subtle and gross does not remain gross for all time. After innumerable "samaya," the subtle changes to gross and gross splits into subtle form. ${ }^{8}$ Similarly, the colour and other attributes of pudgala also change with time. A black colour of one degree can stay in the same condition for a minimum time of one "samaya" and a maximum time of innumerable "samaya." Thereafter, one degree black shall change to up to innumerable degree black by the internal process of "sadguna-hani-vridhhi." Intrinsic modification occurs in every substance every moment. Extrinsic modification of gross aggregates is also certain after innumerable "samaya." So, the paramanu has a dynamic character.

\section{Integration (Bandh)}

All physical matter is produced either by integration (bandh) or by disintegration (bheda) process. The integration is of two types—natural (vaisrasika) and by animate organisms (prayogika). ${ }^{9}$ The natural kind is again of two types - with a definite beginning and without a beginning. Some instances of natural integration, which have a beginning, are clouds, lightening, rainbow, etc.

Integration made by living organisms necessarily has a definite beginning and can be divided into two kinds. ${ }^{10}$

(1) Integration of one kind of matter with another, e.g., production of chemical composites;

(2) Combination of matter with soul in worldly living beings.

The last one is again of two types-karma-bandh, bondage of karma-vargana (with soul), and no-karma-bandh, combination of other groups of pudgala with soul in vital functions and formation of gross body.

Jain philosophy provides elaborate rules for bonding among paramanus. The bonding takes place due to positive and negative charge of paramanus. ${ }^{11}$ The charge of a paramanu varies in a range. Let $\mathrm{q}$ be the minimum indivisible unit charge, positive $\left(\mathrm{q}_{+}\right)$or negative $\left(\mathrm{q}_{-}\right)$, and that the charge increase in multiples of 1,2 or 3, etc. units. The paramanu can have a charge q, 2q, 3q, 4q, etc., q being positive or negative. The rules for bonding between paramanus are given in Table $1 .^{12}$ It is seen that there is some variation in the rules of bonding in Svetambara and Digambara traditions. In both traditions, a paramanu having a minimum charge does not bond with other paramanu. If charge is more than the minimum value and differs by two units or more than the two paramanus can bond according to both traditions. These rules are also applicable to bonding between an aggregate and a paramanu or between two aggregates.

Table 1

Rules for Bonding of Paramanus

\begin{tabular}{|c|c|c|c|c|c|}
\hline & \multirow{2}{*}{$\begin{array}{l}\text { Value of Charge of Two } \\
\text { Paramanus Bonding } \\
\qquad \mathrm{q}_{1}+\mathrm{q}_{2}\end{array}$} & \multicolumn{2}{|c|}{ Svetambara Tradition } & \multicolumn{2}{|c|}{ Digambara Tradition } \\
\hline & & $\begin{array}{l}\text { Similar Charge } \\
\text { Paramanu }\end{array}$ & $\begin{array}{l}\text { Dissimilar Charge } \\
\text { Paramanu }\end{array}$ & $\begin{array}{l}\text { Similar Charge } \\
\text { Paramanu }\end{array}$ & $\begin{array}{l}\text { Dissimilar Charge } \\
\text { Paramanu }\end{array}$ \\
\hline 1 & $q+q$ & No & No & No & No \\
\hline 2 & $q+2 q$ & No & No & No & No \\
\hline 3 & $q+3 q$ & No & No & No & No \\
\hline 4 & $\mathrm{q}+4 \mathrm{q}$ and up to nq & No & No & No & No \\
\hline 5 & $2 q+2 q$ & No & Yes & No & No \\
\hline 6 & $2 q+3 q$ & No & Yes & No & No \\
\hline 7 & $2 q+4 q$ & Yes & Yes & Yes & Yes \\
\hline 8 & $2 q+5 q$ and up to nq & Yes & Yes & Yes & Yes \\
\hline
\end{tabular}


The qualities of the aggregate produced by bonding depend on the qualities of the constituent paramanus or aggregates. For instance, one unit black paramanu on combining with higher degree white paramanu becomes white. When one degree black paramanus combines with one degree white paramanus a grey colour is produced in the aggregate.

\section{Paramanu}

The canonical literature in general and the Bhagwati Sutra in particular define paramanu in various ways from different perspectives. It is the basis (ultimate constituent) of the physical universe. It is indivisible, indestructible, impenetrable, incombustible, and imperceptible to sense organs. ${ }^{13}$ It cannot be split or destroyed by any means whatsoever. It has no half-portion, no middle portion and no pradesa. It has no length, no breadth, and no depth. It is dimensionless. It is truly infinitesimal.

Paramanu is the pure form of pudgala and possesses the intrinsic qualities of touch, taste, smell, and colour. These qualities are attributed to a paramanu for a fundamental reason. It is the basic assumption in Jain philosophy that the fundamental properties of a substance are also eternal; they are neither created nor destroyed. Hence, the basic properties observed in aggregates are also present in paramanu. A paramanu has one of the five primary colours, one of the two smells, one of the five tastes, two of the four primary touch i.e., one either hot or cold and two either smooth (positive change) or rough (negative charge). ${ }^{14}$ Although the four qualities are permanently possessed by a paramanu, the intensity of the qualities does not remain constant. A paramanu possessing one unit of blackness at any moment may sometimes later possess two, three, or many units of blackness. ${ }^{15}$ In the free-state, the mutation is only in the intensities of colour, etc., i.e., $\mathrm{x}$ unit black changes to y unit black but black does not become white or red, etc., however, during and after union with other paramanus change in colour, etc., may also take place. It follows from this that at any given moment there would be paramanus with different intensities of blackness, etc. In the same way, there would be paramanus with various degrees of other qualities.

A single free paramanu is invisible not only to the naked eyes but also to other physical instruments. Its existence is to be inferred by the collective action and reaction of aggregates of infinite paramanus. Only the omniscient (kevalajnani) and those possessing superlative visual intuition (paramavadhi jnani) can perceive and cognize the nature of a free paramanu.

The paramanu is the direct unit of physical substance (pudgala) and also the indirect unit of space, time, and quality magnitude of attributes. ${ }^{16}$ The quantitative and qualitative difference in the various form of the matter (aggregates/pudgala) in space and time domain ultimately depends on the action/reaction of attributes of paramanu. Thus, being the fundamental constituent of physical composite bodies, it may be considered to be the determinant of the difference of aggregates, and for the same reason it is also their substantial cause. By its own motion, it becomes the measure of time unit "samaya."

The paramanus have the innate capacity of uniting with one another to form composite bodies. The composite bodies are liable to the process of disintegration and the united paramanus may become free paramanus and thus the process of association and dissociation goes on eternally. ${ }^{17}$ Paramanu is capable of being dynamically active (kriyavan). When dynamic, it may have spin, vibratory, and migratory motions. ${ }^{18}$ The activity of a paramanu is not continuous, rather it is quanta. The dynamics of paramanu in some respect follows certain rules but it also follows some rules of uncertainty. Paramanu generally cannot be stopped or hindered by any object (apratighati) and at the same time it does not cause hindrance to others. ${ }^{19}$ 
A paramanu in a given space-time domain has various energy states: potential, electro-thermal, kinetic, etc., in view of their basic attributes and their variation as a consequence of change in energy states, which reveals that a paramanu is a vibrating and moving charge. It has also been said that infinite number of paramanus can occupy one space point. ${ }^{20}$ This means that paramanu is bosonic in character. As the paramanu is indivisible, the energy of a paramanu is the smallest amount of energy that can exist in Free State and therefore it can be regarded as energy quanta.

It should be mentioned that the atom described by modern science is not the same as paramanu. The paramanu is weight less (it has infinitesimal energy) and has one colour, one taste, one smell, and two touches whereas an atom has mass and belongs to the type (3) matter; it has five colours, five tastes, two smells, and eight touches. According to Jainism, an atom, rather each of its elementary particles, contains infinite number of paramanus as described below. These paramanus may have positive or negative charge and bond together according to the rules prescribed above. That is, there is bonding between positive and positive, positive and negative, and negative and negative paramanus. The particle formed in this manner has a net charge depending on the majority population of a particularly charged parmanus. For example, an electron has majority population of negative paramanus and a proton has a majority population of positive paramanus. The total negative charge of electrons is equal to the total positive charge of protons in an atom for a stable structure. Jainism does not rule out formation of particles having fractional or multiple charge of electron or proton, but such particles have not been found to form a stable structure and have no practical value.

\section{Vargana (Energy Fields)}

Vargana is an important concept to understand nature particularly at subtle level. Vargana has been defined as pudgala aggregate made up of similar paramanus or as a cluster of paramanus. ${ }^{21}$ There are infinite numbers and types of varganas according to Bhagwati Sutra but eight types are important from the point of view of their association with the soul. ${ }^{22}$ Gommatsara Jivakanda provides another type of classification of varganas on the basis of number of paramanus present in the cluster. ${ }^{23}$ According to this, there are 23 types of main varganas found all over loka. The varganas fall into two broad categories; one has four touches and the other eight touches. The 2nd to 14th order varganas are four-touch type and weight less. The 16th to 23rd order varganas are eight-touch type and have weight. The 15th order vargana falls in between the two categories and its nature is uncertain. ${ }^{24}$

The lower order weight less varganas can be divided in two groups.

(1) Associable varganas — varganas that associate with the soul and form various kinds of subtle bodies and other structures that assist the soul in its worldly functioning;

(2) Non associable varganas—varganas that do not associate with the soul.

The following are the associable varganas.

(1) Ahara vargana. This vargana constitutes the gross, protean (vaikriya), and migratory (aharaka) bodies of organisms;

(2) Fiery (Tejas) Vargana. These vargana constitute the fiery body of organisms;

(3) Sound (Bhasha) vargana. The sound vargana is suitable for producing all kinds of sound including the sound produced by inanimate objects like musical instruments and natural phenomena like thundering of clouds and sound produced by living organisms including speech by humans;

(4) Mind (Mano) Vargana. This vargana constitutes the physical mind (dravya manah) of organisms; 
(5) Karman Vargana. This vargana constitutes the karma bodies of organisms.

The higher order varganas can be divided in three groups.

(1) Varganas that is helpful in formation of (i) gross bodies of plants and (ii) small microorganisms (nigodas, these are regarded as subtle form of vegetation), belonging to category of non-mobile (Sthavara jivas). These varganas assist in formation of plant bodies and bodies of small microorganisms. The vargana that assists in formation of plant body compares with sun light (photons);

(2) Permanent Nil (Sunya) Varganas. Detailed information about these varganas is not available in scriptures;

(3) Gross Matter (Maha skandha) Vargana (GMV). This vargana is supposed to constitute all ordinary matter, visible, and invisible, in the universe including bodies of mobile beings.

The charge in vargana produces an electric field. A moving electric charge in vargana also produces a magnetic field. In view of modern science a field is nothing but a charge in the space-time continuum. All fields, magnetic, electrical, and gravitational, are physical realities. A vargana contains a bundle or packet of energy. The energy density or energy intensity increases with the order of vargana. As mentioned above, varganas of 15th and higher order are supposed to have eight touches i.e., in addition to four basic touches, namely cold, hot, positive and negative charge, other four secondary touches-light, heavy, soft, and hard are also present. These additional touch properties are supposed to come in existence due to bonding between paramanus. The light and heavy touches are supposed to produce the property of weight. In the lower order, varganas of four touches types the paramanus cluster but are not supposed to bond.

The act of bonding between paramanus, interaction, requires energy. When two pramanus bond a part of their energy (potential energy) is used up in bonding reducing the free energy of the vargana that exists as kinetic energy of motion and vibration. Therefore, the maximum velocity of a two-paramanu bonded vargana will be less than the maximum velocity of a single paramanu or a two paramanu unbound vargana. We thus see that lower order varganas having four touches must have higher maximum velocity than eight touches varganas of higher order. The paramanu having two touches has the highest maximum velocity. The lower order vargana are weightless and must be free of gravitational effect. The higher order vargana have gravitational property.

\section{Gross Matter Vargana (GMV) and Matter}

All ordinary matter (visible or invisible) is made up of GMV according to Jain view as mentioned above. We examine now how the sub atomic particles may be produced from GMV. ${ }^{25}$ Consider the case of leptons first. The neutrino is the smallest lepton having negligible mass and no charge. If neutrino is made of GMV, then it must be a combination of at least two GMV, one having positive charge and the other a negative charge. This will be the case when the two GMV have equal and opposite charge. As varganas exist with differing charges, it is very likely that more than two GMV combine to produce a neutral charge in neutrino. So a neutrino of negligible mass should be made up of many GMV. There are three types of neutrinos. The mass of all three types is negligible but still there is a minor difference between them. Such minor variation in mass is obtained by variation in number of GMV in the three types of neutrinos. It may be noted that when the mass of a neutrino is considered to be negligible, the mass of GMV is still less.

Now consider another lepton, the electron. The mass of electron is $0.511 \mathrm{MeV}$, which is millions of times greater than the mass of a neutrino. This means that an electron is made of millions of GMV. In an electron, the 
number of negative charge GMV exceeds the positive charge GMVs giving a net negative charge of $-1.6022 \times 10^{-9}$ coulomb. This also shows that the charge of one GMV is millions of times smaller the charge of an electron. And since a GMV contains infinite paramanus, the quantum charge of a paramanu is really unimaginably small. The lepton muon is more than 200 times heavier, and tau is about 3500 times heavier than electron and therefore, they must contain more GMV in the same proportion.

Next consider the stable baryon particles proton and neutron. These particles are supposed to be made up of quarks. The mass of a proton is 1836.12 times greater than that of the electron and neutron is very slightly heavier than proton. The mass of a quark is uncertain but it is many times more than that of the electron. So a quark is made from that many times more GMV than an electron. There are six types of quarks having fractional charges, both positive and negative, and masses ranging from $2 \mathrm{MeV}$ to $18000 \mathrm{MeV}$. According to Jain view, the fractional charges of quarks are possible by appropriate combination of positive and negative GMV. Another thing we observe is that the charges of up quark, charm quark, and top quark are same but their masses vary considerably. Similar is the case with down quark, strangeness quark, and bottom quark. Formation of these quarks is clearly possible with suitable combination of GMV. So, in Jain view, quarks and leptons are composite particles having property of gravity. Many more types of particles can be formed, including those not discovered so far.

Mass of matter is nothing but transformation of energy, that is, both matter and energy are but two modifications of a single principle, has been only recently realized in science. Jain physics has identified all forms of matter and energy as modification of the same substance pudgala. Intra-convertibility of various forms of energy - mechanical into electrical, electrical into heat, light, sound, etc.-which is the basis of modern technology — has been recognized by Jain philosophers as the basic attributes of pudgala, since all forms of energy are fundamentally the modification of the same substance, paramanu pudgala.

\section{Dark Energy and Dark Matter}

There is no direct mention of dark energy and dark matter in Jain scriptures. The existence of dark energy in science has been postulated to satisfy the condition of expanding and accelerating universe and it is supposed to have anti gravity property. The non associable varganas described above are weight less and gravity free. These varganas may comprise a good fraction of the total mass present in the loka that is gravity free but do not possess anti gravity property as postulated by modern science. The Permanent Nil Varganas may be considered to constitute the dark matter as they are not detected by ordinary means. These varganas may constitute a significant proportion of mass present in the loka. The matter formed by these varganas could be non-baryonic as the baryonic matter is formed by higher Gross Matter Vargana (GMV). Jainism supports the scientific view that mass is not the exclusive property of ordinary matter. According to Jainism, even photons have mass.

Jain scriptures mention about bodies on which even beings having great powers, e.g., heavenly beings fear to go. Jain canon Bhagwati Sutra describes existence of dark structures in space comparable to black holes. These structures are of two types, one Tamaskaya, Mass of Darkness, and two Krsnaraji, Black Streaks. ${ }^{26}$ Both are pitch dark structures containing no parts like stars, sun, moon, planet, etc., and no life. Both have rains meaning thereby that they attract neighbouring matter that appears as showers on the surface. The light of other stars and moons becomes dim as they approach these structures. Tamaskaya is a huge structure extending from a location far away from Jambudweepa, supposed to be our Earth, and going up to fifth heaven in the upper loka. This is said to have been formed by transformation of water bodies of organisms and other matter. The 
Krsnaraji, eight in number in a closed loop structure located in fifth heaven in upper loka, is said to have been formed by transformation of earth (bodied beings) and other matter. This indicates that dark holes (or dark matter) can be formed in two ways one from water source i.e., fluidic matter and the other from earth like solid matter.

\section{The Laws of Subtle Cosmos}

Modern science has discovered that as we go down from the macro to the micro state of matter, new attributes of matter come in action and the number of attributes increase. The macro world is deterministic and follows the laws of classical mechanics. The micro world follows the laws of quantum mechanics. Some laws of classical mechanics are not valid in the micro world. It may be noted that macro and micro world of science are comprised of eight-touch gross aggregates containing mass, which consists of higher-order varganas in bonded form. The weight less four-touch vargana subtle aggregates which exist only in energy form is a different class of matter. The weight less, four-touch varganas do not carry the fundamental forces as their paramanus are supposed to be in unbound state. Their behavior, therefore, must not be governed by known laws of science. On extrapolating, we expect that at subtle level of the physical world, e.g., weight less four-touch vargana, there may be yet another set of principles in operation, which is still not discovered by science.

\section{Organic and Inorganic Matter}

The pudgala can be classified into three types in respect of the cause of transformation ${ }^{27}$ viz.

(1) Prayoga-Parinata (living organic matter). The pudgala (matter), which is taken in and transformed into body form by vital processes of living beings, falls in this category;

(2) Misra-parinata (past living matter, dead organic). The pudgala (matter), which was associated with living beings in the past, but is now abandoned by it, and therefore, is no longer being transformed by the agency of vital processes, but undergoes self-transformation, is misra (mixed)-parinata. Shoe-leather, meat, etc., are instances of this type;

(3) Visrasa-parinat (non-living matter, inorganic). Matter, which undergoes natural transformation i.e., without interaction with living beings, is visrasa-parinat. Clouds, rainbow, meteors, etc., are instances of this class.

Subtle changes take place in every substance every moment. Gross changes occur in soul and pudgala only. In this respect, both the soul and pudgala are similar but as far as the total changes are concerned pudgala far exceeds soul. Changes in pudgala make the world change. Everything from the beginning to end in the world is governed by the natural changes taking place in pudgala and soul. The universe is self-managed from this point of view. Considering alienated modifications, the universe is governed by changes caused by union and separation of soul and pudgala. Soul and pudgala influence each other and both experience self-generated and alienated modification.

The living matter exhibits some properties that are not found in non-living inorganic matter. Modern science does not differentiate between these two types of matter and considers them to be made up of the chemical elements and applies similar rules to both. Jain philosophy says that living body is a combination of soul and pudgala and exhibits the properties of both the components and just not the chemical elements. For example, a living body shows the property of consciousness which is the property of the soul and not of matter. 
Modern science is trying hard to explain consciousness as an emergent property, reducible to the properties of matter, and this has become a subject of controversy even amongst the scientists. The behavior of body parts is also influenced by soul through karma. For example, the behavior of genes cannot be explained purely on the basis of material properties; they are also influenced by karma. Thus, complete explanation of behavior of organisms needs consideration of existence of both the soul and the matter.

Taking a comparative view, the amount of prayog-parinat (body matter) is least of all, the misra — parinat pudgala is infinite times more and the visrasa — parinat pudgal is still infinite times more than this.

\section{Motion}

Two types of motions are described in Jainism. ${ }^{28}$

(1) Sparshad gati-motion under the action of touch properties. The moving object makes contact with other matter present on space points. This applies to the motion of pudgala;

(2) Asparshad gati-motion without the action of touch properties. The moving object does not make contact with other matter present on space points. This applies to the motion of pure soul.

We know there are eight touch properties. These are divided in four groups.

(1) Heat—cold and hot touch;

(2) Electric charge—snigdha and ruksa touch;

(3) Stress/strain—mridu and karkash touch;

(4) Gravity (weight)—light and heavy touch.

This means that motion may take place due to (1) heat, e.g., convection currents in fluids, (2) electric force (and also magnetic force), e.g., propagation of light and radiations, electric motor, etc., (3) stress and strain, e.g., stretching of solids, viscous action in liquids, etc., and (4) gravity, e.g., motion of falling objects, motion of astronomical objects, etc. The asparshad gati does not involve any of these forces.

We now consider motion of different types of pudgala objects.

(1) Motion of a paramanu

A paramanu is apratigati, in free state, it does not interact with any other object. Hence, there is no external influence of any kind on the motion of a paramanu. A paramanu moves due to its intrinsic characteristic of dynamic action. Its motion is hindered only when it collides with another paramanu, which is a very rare possibility. It may be noted that the laws of motion of science and the limit imposed by special theory of relativity do not apply to paramanu as the forces on which these laws and theories are based are absent in this case. The dynamic activity of a paramanu is uncertain as described before and it may move with low, medium, or high velocity as determined by the property of sadgun-hani-vridhi. In the extreme case of highest velocity, the paramanu can travel from one end to another end of loka in one samaya if not hindered by another paramanu.

(2) Motion of four-touch Vargana

A four-touch vargana may contain two to infinite number of paramanus. The fundamental forces, recognized by science, are still absent in this type of vargana and its motion is not governed by known laws of science and the special theory of relativity. However, there is affinity between paramanus in a vargana and so the maximum velocity of this type of vargana would be less than the maximum velocity of a paramanu as explained above. Due to large number of paramanus, the chances of its colliding with other vargana are 
significant. On collision, the two varganas may merge and form a bigger vargana of the same kind or a vargana of another kind.

(3) Motion of eight-touch Vargana

Eight-touch vargana contains paramanus in the bound state and all the fundamental forces must be present in it. All electromagnetic radiations fall in this category. This eight-touch type of varganas, therefore, is expected to obey the known laws of science and the limit on speed imposed by the special theory of relativity may apply. On account of small mass, the gravitational force must be negligible and the motion is largely governed by electromagnetic force, e.g., in the case of a photon and small microorganisms (nigodas).

(4) Motion of particles (made of Mahaskandh Vargana)

In the case of matter formed of GMV, at the level of subatomic particles and atoms, the gravitational force is still very small and other forces determine the motion. As the aggregates grow in size, the gravitational force increases and the effect of electromagnetic force diminishes because the number of parmanus having positive and negative charge in the aggregate is likely to be of the same order canceling the effect of each other. Thus, the motion of large particles and objects is governed mainly by the gravitational force.

\section{Conclusions}

The paramanu of Jain philosophy is the smallest indivisible fraction, quantum, of energy. The Jain paramanu, the real energy quanta, is far too smaller than the quantum of energy, photon, presumed by science. Science has discovered particles like quark but it still remains a mystery what makes the quark. The journey of science has been from gross to fine and it has gone to the level of quark. Jain philosophy starts from the ultimate particle paramanu and proceeds up to the gross form of matter. Jain philosophy says that the fundamental constituent of nature is energy and paramanu is its ultimate unit. Paramanu makes up vargana and vargana make up photon and the gross particles like quark, electron, etc. Jain philosophy presents the subtler form of matter which science has not discovered so far. The story of matter from quark and electron onward is known to science. Jain philosophy also offers some plausible explanation to puzzling questions like what is the nature of matter other than the ordinary matter postulated by science. Thus, Jain philosophy and science together reveal more complete picture of the physical reality.

\section{Notes}

\footnotetext{
1. Acharya Tulsi, Acharya Mahaprajna, “Sthanang Sutra,” Jain Vishva Bharati, 5.174.

2. Acharya Tulsi, Acharya Mahaprajna, “Bhagwai,” Jain Vishva Bharati, 20.5.1.

3. Ibid., 12.118.

4. Acharya Tulsi, Acharya Mahaprajna, “Sthanang Sutra,” Jain Vishva Bharati, 4.135.

5. Acharya Jai, "Bhagwati Joda," Jain Vishva Bharati, 18.6.117.

6. Acharya Abhaya Dev, "Bhagwati Vriti,” Agamodaya Samiti, 1.393.

7. Acharya Tulsi, Acharya Mahaprajna, “Bhagwai,” Jain Vishva Bharati, 5.169.

8. Acharya Mahaprajna, “Jain Darshan aur Anekanta,” Adarsh Sahitya Sangh, 69.

9. Acharya Akalanka, “Tatvartha Rajvartik,” Bhartiya Jnanapith, 5.24.1.485.

10. Ibid., 5.24.9.487.

11. Acharya Umaswati, “Tatvartha Sutra,” Hindu Vishva Vidyalaya, 5.33.

12. Ibid., 5.34-37. Acharya Akalanka, “Tatvartha Rajvartik,” Bhartiya Jnanapith, 5.37.2.499-500.

13. Acharya Tulsi, Acharya Mahaprajna, "Bhagwai,” Jain Vishva Bharati, 5.154.1.

14. Shantyacharya, "Uttaradhyan Vriti,” Devchand Lalbhai Jain, 24.

15. Acharya Tulsi, Acharya Mahaprajna, “Bhagwai,” Jain Vishva Bharati, 25.350.
} 
16. Ibid., 20.37-41.

17. Ibid., 5.169 .

18. Ibid., 5.150.

19. Muni Nagraj, “Jain Darshan aur Adhunik Vigyan,” Atmaram and Sons, 37.

20. Acharya Pujyapada, “Sarvarth Siddhi,” Bhartiya Jnanapith, 5.14, 5.16.

21. Acharya Malayagiri, “Avashyak Vriti,” Jain Vishva Bharati, 278.

22. Acharya Tulsi, Acharya Mahaprajna, “Bhagwai,” Jain Vishva Bharati, 2.4.

23. Acharya Nemichandra Siddhant Chakravarti, “Gommatsara Jivakanda,” Bhartiya Jnanapith, 594-5.

24. Kachhara, N. L., "Jain Metaphysics and Science: A Comparison,” Prakrit Bharati Academy, 109-58.

25. Kachhara, N. L., "Scientific Explorations of Jain Doctrines,” Motilal Banarasidass, 189-92.

26. Acharya Tulsi, Acharya Mahaprajna, "Bhagwai,” Jain Vishva Bharati, 6.70-6.118.

27. Acharya Tulsi, Acharya Mahaprajna, "Sthanang Sutra,” Jain Vishva Bharati, gatha 401.

28. Acharya Tulsi, Acharya Mahaprajna, “Sthanang Sutra,” Jain Vishva Bharati, 965. 